\title{
From Mesmer to animal magnetism
}

Over the years, many examples of sensory systems that humans apparently do not possess have popped up in interesting places among the vertebrates. Some are extensions of known senses echolocation in bats and whales, infrasound and ultrasound detection in birds, and the sexpheromone receptors (the vomeronasal system) in the nose of most higher vertebrates. Others, such as the electroreceptive organs of sharks and rays, and the infrared detectors of snakes, depend on highly specialized receptor cells. These discoveries generated little scientific controversy.

Not so biomagnetism, which in the late eighteenth century received an early bad rap from the activities of Franz Anton Mesmer and his followers. The mesmerites claimed that they could cure disease by exposing patients to magnetized objects, a claim debunked by a commission (which included Benjamin Franklin) appointed by King Louis XVI. The subject then fell into a long period of disrepute until the 1940s, when experiments suggesting that pigeons might use geomagnetic cues during homing generated great interest. These, however, were difficult to reproduce; likewise, conditioning experiments designed to elicit magnetoreception in the laboratory also failed.

The main stumbling block was in seeing how a geomagnetic stimulus could be converted into a signal that an individual cell could detect. Backof-the-envelope calculations ruled out most of the obvious methods (paramagnetism, electrical induction, the Hall effect, nuclear magnetic resonance). The simplest strategy - that of having a small permanent magnet - was dismissed on the grounds that there were no physiological ferromagnetic materials ${ }^{2}$ As discussed in the main text, the discovery of magnetite eventually knocked that argument on the head.

Meantime, behavioural experiments kept on coming up with apparent geomagnetic effects on animal behaviour. But it took nearly two decades to realize that the geomagnetic compass used by adult birds was programmed to be ignored if other orientation cues (such as a Sun or star compass, polarized skylight, infrasound and ultrasound) were present. These orientation cues constitute a complex but consistent web of interacting responses, which are used not only by birds but in all major vertebrate groups and many invertebrates (reviewed in ref. 5).

J.L.K. questions as threshold sensitivities and frequency response, as has been done with similar conditioning experiments with honeybees ${ }^{6}$.

Second, and even more excitingly, they have traced the sensory nerves back to possible magnetoreceptor cells. After confirming that the ophthalmic branch of the trigeminal nerve in fish contains magnetically receptive fibres, as it does in birds ${ }^{7}$, they used a lipidtracing dye to map the fibres back to the brain and to the location of putative receptor cells. Attempts to trace the avian magnetoreceptive nerves have failed because of the problem of identifying magnetite crystals in optical sections. Walker and colleagues' application of confocal laser microscopy provided the techniques for both tracing individual neurons back through a complex three-dimensional path and, by calibrating the confocal reflections with magnetotactic bacteria, for identifying possible magnetite crystals in the target cells.

Note, however, that the iron oxide mineral has not yet been identified conclusively. The cells containing the confocal reflections have distinctive shapes and always lie within a discrete sublayer of the olfactory lamellae (at the tips, near the distal terminals of fine branches of the trigeminal nerve), and the particles have similar size and shape to those extracted from salmon ${ }^{8}$ (Fig. 3). But followup studies with conventional transmission electron microscopy and electron diffraction are required to confirm that the iron oxide is indeed magnetite.

A huge range of organisms can sense magnetic fields ${ }^{5}$. Do humans remain an exception? We certainly have a trigeminal nerve, with an ophthalmic branch, and we can also make biogenic magnetite. At least one other vertebrate sensory system thought to have been lost in the final stages of human evolution — the sex-pheromone-sensing vomeronasal organ - has recently been found to be both present and functional ${ }^{9}$ (human vomeronasalins now form a booming perfume industry). Other effects, such as the ability of a 1-millitesla static field to elicit epileptiform activity in patients preparing for brain surgery ${ }^{10}$, have also emerged. Finally, some humans, particularly Polynesian navigators, seem able to judge direction in the absence of all obvious cues (Sun, Moon, stars, waves and so on $)^{11}$. So there is hope for our lost canoeist - the final word on the existence of human magnetoreception has certainly not yet been written.

Joseph L. Kirschvink is in the Division of Geological and Planetary Sciences, California Institute of Technology, Pasadena, California 91125, USA.

1. Walker, M. M. et al. Nature 390, 371-376 (1997).

2. Griffin, D. R. Q. Rev. Biol. 19, 15-31 (1944).

3. Kirschvink, J. L., Jones, D. S. \& McFadden, B. J. Magnetite Biomineralization and Magnetoreception in Organisms: A New Biomagnetism (Plenum, New York, 1985).

4. Kirschvink, J. L., Kobayashi-Kirschvink, A. \& Woodford, B. J. Proc. Natl Acad. Sci. USA 89, 7683-7687 (1992).

5. Wiltschko, R. \& Wiltschko, W. Magnetic Orientation in Animals (Springer, Berlin, 1995).

6. Kirschvink, J. L., Padmanabha, S., Boyce, C. K. \& Oglesby, J. J. Exp. Biol. 200, 1363-1368 (1997).

7. Beason, R. C. \& Semm, P. J. Exp. Biol. 199, 1241-1244 (1996)

8. Mann, S., Sparks, N. H. C., Walker, M. M. \& Kirschvink, J. L. J. Exp. Biol. 140, 35-49 (1988)

9. Berliner, D. L., Monti-Bloch, L., Jennings-White, C. \& Diaz-Sanchez, V. J. Steroid Biochem. Mol. Biol. 58, 259-265 (1996).

10. Fuller, M., Dobson, J., Gregor-Wieser, H. \& Moser, S. Brain Res. Bull. 36, 155-159 (1995).

11. Finny, B. Curr. Anthropol. 36, 500-505 (1995).

\section{The big splash}

\section{Jan Smit}

S eventy per cent of the Earth is covered with sea water, and most of that is ocean, so most of the large asteroids and comets striking the planet should hit deep water. But very little remains of these impacts - virtually all evidence of large impacts, in the form of craters and associated debris, is found on land. On page 357 of this issue ${ }^{1}$, Gersonde et al. describe the evidence for and consequences of the impact of an asteroid, $1-4 \mathrm{~km}$ in diameter, in a 5,000-mdeep ocean basin, 1,500 $\mathrm{km}$ southwest of Chile. The asteroid hit the Earth in the late Pliocene, some 2.15 million years ago.

The new results come from three piston cores, taken in the course of the 1995 FS
Polarstern expedition to the Bellingshausen Sea in the Southern Ocean. This expedition was dedicated to finding more remains of this, the only known oceanic impact, because piston cores, taken 30 years ago in the same area by the USNV Eltanin, yielded the iridium anomaly typically associated with extraterrestrial impacts, and melted and unmelted meteoritic debris. This debris identified the impactor as an asteroid, a basaltic achondrite which was named the Eltanin meteorite. But many questions remained.

The Polarstern cores were taken in different depths of water - one on a seamount (depth 2,707 m), one at intermediate depth 
$(3,965 \mathrm{~m})$ and one on the Bellingshausen abyssal plain $(4,961 \mathrm{~m})$. All three yielded a complete sedimentary sequence across the impact 'horizon', although only the core at intermediate depth penetrated through the entire sequence. The cores all reveal a similar sequence, one that is consistent with an oceanic impact and bears a strong resemblance to the sequences found in the Gulf of Mexico that were deposited as a result of the Chicxulub impact on the Yucatán, Mexico, 65 million years ago.

A crater has not been found, however, and together with the absence of silicate debris on the ocean floor, that implies that the impact never penetrated into the basaltic basement of the ocean floor. From work with early impact models it seemed that a small $(<1-\mathrm{km})$ asteroid could penetrate to the sea floor and leave a crater, but newer models ${ }^{2}$ support the conclusion that the Eltanin bolide may have been as large as $4 \mathrm{~km}$ without actually striking the sea floor. This is probably part of the reason that craters have thus far not been found on the deep ocean floor.

Nonetheless, the new cores show that the impact must have been substantial and disturbed the sea floor over hundreds of kilometres. Extremely strong currents resulting from the opening and closing of the transient 'oceanic' crater removed large amounts of sediments as 'rip-up clasts' and deposited them elsewhere. Gersonde et al. speculate that some sediments were probably blown away from the sea floor and were globally dispersed as ballistic ejecta. It seems that these may have landed on the Transantarctic Mountains, providing a nice third alternative explanation for the origin of some puzzling diatom-bearing deposits that were earlier explained as wind-blown dust or glacial till deposits ${ }^{3}$.

The Polarstern core sequences show a threefold subdivision of sedimentary units wedged between packages of undisturbed

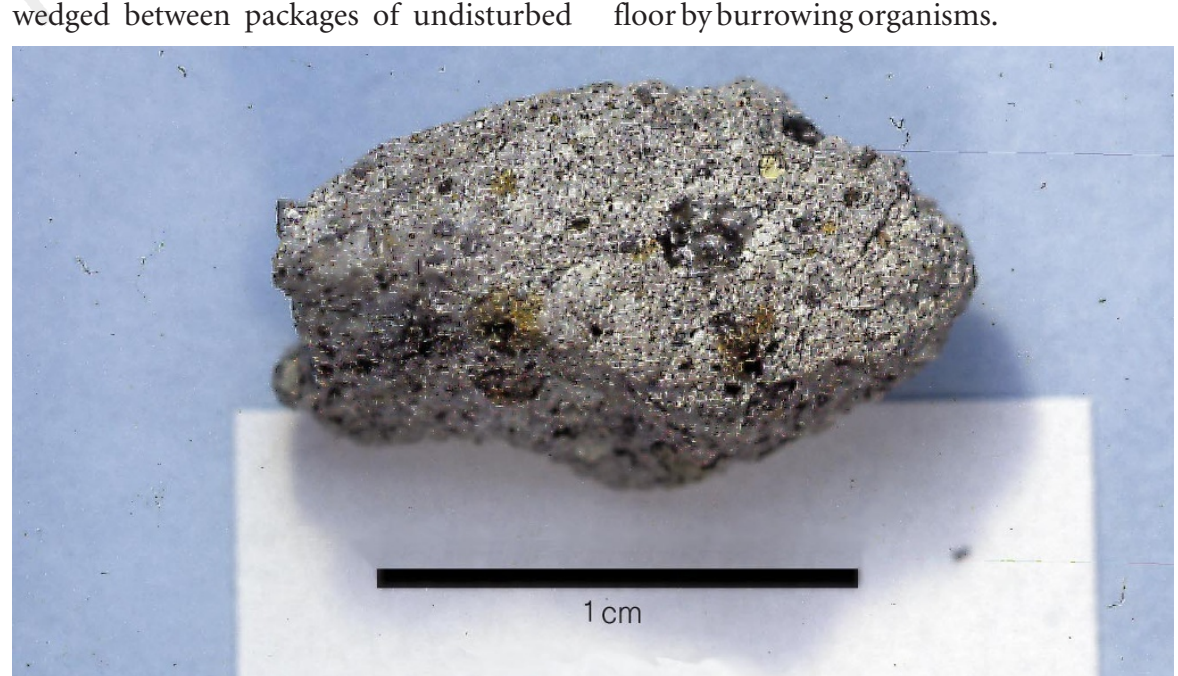

Figure 1 The largest unmelted remnant of the Eltanin meteorite recovered by FS Polarstern. It is a fragment of mesosiderite, a rare class of differentiated, stony-iron meteorite, and comes from core PS2708-1 (that drilled at intermediate depth; see Fig. 3 on page 359). (Photo courtesy of R. Gersonde.) (see Fig. 3 on page 359). The lowermost unit consists of large blocks and slumped material that was swept along by the violent currents initiated by the impact. Because the cores were taken at different depths, it is possible to identify the blocks' origins. On the seamount, lumps of clay originating from the abyssal plain below the carbonate compensation depth (below which almost all carbonate shells dissolve, and only carbonate-free clays are deposited) were found well above that depth. Conversely, blocks of calcareous sediments from the seamount were swept into the abyssal basin. This lowermost unit has been traced in surveys over a wide area as a seismically transparent unit, meaning that it is not a layered but an acoustically uniform sediment. It fills topographic lows to a depth of up to $60 \mathrm{~m}$ and extends over hundreds of kilometres.

The overlying second unit consists of a laminated, grain-size graded sand. The laminae indicate deposition by currents, although not as strong as those involved in the basal unit; the grading indicates settling from suspension. Apparently, the ocean was still in motion at this stage, as a consequence of the impact, but currents were not strong enough to erode sediments away or prevent sedimentation of smaller particles. The top of this second unit contains the first, coarse, meteoritic debris (Fig. 1), which landed on the sea floor after a journey through space, the atmosphere and the water column, taking a minimum of four hours.

The third uppermost unit represents the slow deposition of fine, suspended material. Residual currents were then too weak to move particles over the sea floor, except in the layered basal part of the unit which is strongly enriched in meteoritic debris. The settling of such fine material may have lasted for weeks, but the process was still fast enough to prevent colonization of the sea floor by burrowing organisms.

This threefold sequence is strikingly sim- 